\title{
Coding of Electronic Laboratory Reports for Biosurveillance, Selected United States Hospitals, 2011
}

\author{
Sanjaya Dhakal ${ }^{1 *}$, Sherry L. Burrer ${ }^{2}$, Carla A. Winston ${ }^{3}$, Achintya Dey $^{1}$, Umed Ajani ${ }^{1}$, Samuel L. \\ Groseclose $^{4}$
}

1. Division of Health Informatics and Surveillance, Center for Surveillance, Epidemiology and Laboratory Services, Office of Public Health Scientific Services

2. Division of Environmental Hazards and Health Effects, Injury and Environmental Health, National Center for Environmental Health, Office of Non-communicable Diseases

3. Veterans Health Administration, Office of Public Health, Office of Public Health Surveillance and Research, U.S. Department of Veterans Affairs

4. Office of Science and Public Health Practice, Office of Public Health Preparedness and Response

\begin{abstract}
Objective: Electronic laboratory reporting has been promoted as a public health priority. The Office of the U.S. National Coordinator for Health Information Technology has endorsed two coding systems: Logical Observation Identifiers Names and Codes (LOINC) for laboratory test orders and Systemized Nomenclature of Medicine-Clinical Terms (SNOMED CT) for test results.

Materials and Methods: We examined LOINC and SNOMED CT code use in electronic laboratory data reported in 2011 by 63 non-federal hospitals to BioSense electronic syndromic surveillance system. We analyzed the frequencies, characteristics, and code concepts of test orders and results.

Results: A total of $14,028,774$ laboratory test orders or results were reported. No test orders used SNOMED CT codes. To describe test orders, $77 \%$ used a LOINC code, $17 \%$ had no value, and $6 \%$ had a non-informative value, "OTH". Thirty-three percent (33\%) of test results had missing or non-informative codes. For test results with at least one informative value, $91.8 \%$ had only LOINC codes, $0.7 \%$ had only SNOMED codes, and $7.4 \%$ had both. Of 108 SNOMED CT codes reported without LOINC codes, $45 \%$ could be matched to at least one LOINC code.

Conclusion: Missing or non-informative codes comprised almost a quarter of laboratory test orders and a third of test results reported to BioSense by non-federal hospitals. Use of LOINC codes for laboratory test results was more common than use of SNOMED CT. Complete and standardized coding could improve the usefulness of laboratory data for public health surveillance and response.
\end{abstract}

Keywords: Logical Observation Identifiers Names and Codes (LOINC); Systemized Nomenclature of Medicine Clinical Terms (SNOMED CT); electronic laboratory report; computerized medical record systems; biosurveillance

Correspondence: $\underline{\text { hgj2@cdc.gov }}$

DOI: $10.5210 /$ ojphi.v7i2.5859

Copyright @2015 the author(s)

This is an Open Access article. Authors own copyright of their articles appearing in the Online Journal of Public Health Informatics. Readers may copy articles without permission of the copyright owner(s), as long as the author and OJPHI are acknowledged in the copy and the copy is used for educational, not-for-profit purposes 


\section{Introduction}

Because Electronic Laboratory Reporting (ELR) has been seen to be more accurate, timely, and costeffective than reporting by other conventional means (e.g., mail, fax, etc.), ELR adoption has been systematically promoted as a public health priority [1-7]. A major deterrent to laboratory data being used in public health research and biosurveillance programs seems to be the lack of interoperability of automated laboratory information management systems [8-12]. Standardized, universal coding that records laboratory test order and result information in a structured and systematic fashion is an essential component of interoperable ELR systems $[9,13,14]$. The use of local codes or terminology and unstructured text fields to describe laboratory test orders and results varies widely among laboratories $[15,16]$. Several coding strategies are available to make electronic laboratory data more computational and thus readily interchangeable electronically. Long established coding systems such as International Classification of Diseases (ICD) and Current Procedural Terminology (CPT) are used for insurance reimbursement and other administrative purposes. ICD codes are primarily designed for classifying diseases and other health conditions while CPT is designed to record a medical service or a procedure. Therefore, coding laboratory information is out of scope for ICD and CPT coding systems. Several coding strategies are available to make electronic laboratory data more computational and thus readily interchangeable electronically; Logical Observation Identifiers Names and Codes (LOINC) and Systemized Nomenclature of Medicine Clinical Terms (SNOMED CT) are the two most comprehensive coding systems representing lab test type and result information.

Therefore, these two information coding systems were specifically recommended for use in coding laboratory information in electronic health records by the U.S. Department of Health and Human Services Office of National Coordinator for Health Information Technology

Established in 1994, LOINC is a coding system designed to identify medical laboratory observations and procedures. LOINC covers both laboratory tests and clinical observations enabling coding of test orders and test results. The system has been endorsed by the American Clinical Laboratory Association and the College of American Pathologists. A LOINC code is composed of six attributes that characterize the details of laboratory test orders and results, namely: component (e.g., Escherichia coli, potassium), property (e.g., arbitrary concentration, mass concentration), timing (e.g., point in time, over a span of time), system (e.g., stool, blood), scale (e.g., ordinal, nominal), and method (e.g., culture, microscopy). The most recent version of the LOINC database contains more than 70,000 codes [17-22]. LOINC codes are updated twice a year to reflect changes in diagnostic practices over time.

Established in 1965, SNOMED CT is a coding system designed to identify anatomic and clinical pathology information and laboratory results. The system is a collection of medical terms, codes, findings and procedures. A SNOMED CT code is composed of the following three attributes; unique concepts (e.g., E. coli), concept descriptions (e.g., is a), and relationships between concepts (e.g., bacteria present in stool). The system is updated twice a year and currently includes over 300,000 unique concepts [23]. SNOMED CT is the ontological basis of the upcoming International Classification of Diseases $11^{\text {th }}$ Edition (ICD-11) revision spearheaded by the World Health Organization [24,25].

\section{Objective}

We examined the use of LOINC and SNOMED CT codes for coding laboratory test orders and results in laboratory reports transmitted to BioSense program from 63 non-federal hospitals in the calendar year 2011. In this report, we present the first national level description of the use of LOINC and SNOMED 
CT codes from biosurveillance data to characterize laboratory test orders and results reported by hospital-based laboratories.

\section{Materials and Methods}

BioSense is a syndromic surveillance system supported by the U.S. Centers for Disease Control and Prevention (CDC), and it receives daily medical encounter data from participating hospitals. During 2011, 11\% of participating non-federal hospitals also shared laboratory data with BioSense [26,27]. Details of the data transmission from hospitals to CDC's BioSense program have been previously described [28-30]. In brief, data reporting standards follow guidance from the Public Health Information Network Messaging System's (PHINMS) syndromic surveillance message guide and are transmitted securely via digital certificates and data encryption [31]. The laboratory reports included in this analysis were transmitted using version 2.3.1 of Health Level 7 (HL7) message formats. All laboratory reports from non-federal hospitals reporting to BioSense from January 1, 2011 through December 31, 2011 were extracted from HL7 messages and converted to SAS analytic data files. Reports included laboratory test orders only, test orders with results, and results only.

Data elements in each laboratory message can be broadly categorized into three sections: 1) administrative data elements, 2) laboratory test order related data elements, and 3) laboratory result related data elements. Administrative data elements include date of visit, type of service facility, testing laboratory ID, and date of HL7 message creation. Laboratory test order data elements include order number, order test name, order test codes (local and "standard"), order test coding system, and segments of coding structure. Laboratory result data elements are grouped into two categories: observation identifier (OBR) and observation value (OBX). Elements of OBR include observation result code, observation result coding system, local observation result code, observation result text (local and "standard"), and observation result type. Elements of OBX include result coding system, result test code (local and "standard"), result status, result notes, result test date, result test name (local and "standard"), result unit, test interpretation, and details of the sample to be tested (component, property, timing, system, scale, method). Additionally, BioSense laboratory message data include site and type of the specimen, diagnostic criteria, and test sequence number.

Each laboratory test result with result code was categorized into one of six result status categories, namely: "final result”, "preliminary result”, "specimen in the laboratory”, “correction”, "deletes OBX record", and "result can't be obtained for this observation". Since results identified as "preliminary" constituted almost one-third of all laboratory results reported we analyzed all reported results regardless of their status. Later we compared our analysis with findings from a separate review limited to "final" results.

We analyzed the frequency distribution of laboratory test orders and results to determine characteristics of the test orders and results reported, and to examine the 25 most common test orders and results. Unique LOINC or SNOMED CT codes used in the reports were identified, along with the percentage of reports that were missing a standardized code. For each of the SNOMED CT codes reported without corresponding LOINC codes, we used the Public Health Information Network Vocabulary Access and Distribution System (https://phinvads.cdc.gov) web sites to obtain the SNOMED CT concept or descriptive text. Then each concept was searched in Regenestrief LOINC Mapping Assistant (RELMA ${ }^{\circledR}$ ) software version 5.8, to determine if there was a LOINC code that might corresponded to the SNOMED CT concept. Since the value "OTH” in HL7 messages does not clearly indicate a specific laboratory test order or results, rather it indicates concepts not represented by the code system; we 
treated reports coded with "OTH" as non-informative data. This analysis was determined to be a public health surveillance activity rather than human subject research requiring institutional board review.

\section{Results}

Out of 569 non-federal hospitals reporting data to BioSense in 2011, 63 (11.1\%) hospitals in 14 states submitted 14,028,774 laboratory reports (test orders and/ or results) from 821,108 unique laboratory visits. On average, 38,000 laboratory reports were reported daily. In the reports with at least one result code, the hospital diagnostic service ordering the test was categorized into five groups: microbiology (94.7\%), serology (2.8\%), outside laboratory (1.7\%), virology (0.5\%), and immunology (0.4\%).The reports consisted of final (68.7\%), preliminary (31.0\%), specimen in the lab (0.2\%) and corrected $(0.1 \%)$ result status (Table 1).

Table 1. Characteristics of hospital-based laboratory data reported to BioSense, 2011

\begin{tabular}{|l|l|}
\hline Characteristics & N (\%) \\
\hline Number of reporting hospital laboratories & $63(100)$ \\
\hline Number of unique laboratory visits & $821,108(100)$ \\
\hline \begin{tabular}{|} 
Number of laboratory reports (test orders or test results) \\
LOINC order codes
\end{tabular} & $14,028,774(100)$ \\
Non-informative order codes (missing or HL7 null & $10,776,494(76.8)$ \\
code "OTH”) & $3,252,280(23.2)$ \\
\hline Number of laboratory reports with test results code & $9,347,179(66.6)$ \\
LOINC or SNOMED CT or both result codes & $4,681,595(33.4)$ \\
Non-informative codes (both missing or one missing & \\
and the other is HL7 null code “OTH”) & \\
\hline Number of test results with code by code system & $9,347,179(100)$ \\
LOINC codes only & $8,584,826(91.8)$ \\
SNOMED CT codes only & $69,566(0.7)$ \\
LOINC and SNOMED CT both & $692,787(7.4)$ \\
\hline Number of unique LOINC codes for orders & $805(100)$ \\
\hline Number of unique codes for results & $608(100)$ \\
SNOMED CT codes & $1,428(100)$ \\
LOINC codes & \\
\hline Test result status & $9,347,179(100)$ \\
Final result & $6,420,538(68.7)$ \\
Preliminary result & $2,898,975(31.0)$ \\
Specimen in lab & $18,739(0.2)$ \\
Correction & $7,011(0.1)$ \\
Deletes observation value (OBX) record* & $331(0.0)$ \\
Result can’t be obtained for this observation & $7(0.0)$ \\
\hline Diagnostic services & $9,347,179(100)$ \\
Microbiology & $8,849,051$ \\
Serology & 258740 \\
Outside Laboratory & $161,561(1.7)$ \\
Virology & $42,974(0.5)$ \\
Immunology & $34,853(0.4)$ \\
\hline
\end{tabular}


Among the 14,028,774 laboratory test reports, 76.8\% had LOINC order codes, while for the rest either the codes were missing (16.8\%) or had the HL7 null code, "OTH" (6.4\%). No test orders were reported using a SNOMED CT code. Out of 10,776,494 laboratory test orders with LOINC codes, 9,347,179 (86.7\%) also had either LOINC or SNOMED CT or both codes for the test result.

Of all laboratory reports, 9,347,179 (66.6\%) had at least one result code (LOINC or SNOMED CT or both); of these, $91.8 \%$ had only LOINC codes, $0.7 \%$ had only SNOMED CT codes while 7.4\% had both codes (Table 1). Of the remaining 4,681,595 laboratory reports, 63.4\% were missing a SNOMED or LOINC result code with "OTH" reported as a result value while $36.6 \%$ had neither LOINC nor SNOMED codes for the results. Laboratory test results with "final" status comprised the majority $(68.7 \%)$ of all laboratory reports with result codes (Table 1) Findings from the analysis comparing results with "final" status to all results were comparable except that the number of laboratory test results with only LOINC codes differed (91.8\% in all messages versus $61.4 \%$ in only "final" result messages). This suggests that results reported as "preliminary" were not updated when result status changed to "final".

Table 2.Twenty five most common hospital-based laboratory order test types reported to BioSense, 2011, with Logical Observation Identifiers Names and Codes (LOINC) codes

\begin{tabular}{|l|l|l|}
\hline LOINC Test Name & $\begin{array}{l}\text { LOINC } \\
\text { Code }\end{array}$ & Test Orders N (\%) \\
\hline Bacteria identified (Blood) & $600-7$ & $3770050(35.0)$ \\
\hline Bacteria identified (Urine) & $630-4$ & $1637400(15.2)$ \\
\hline Bacteria identified- Respiratory culture & $32355-0$ & $522495(4.8)$ \\
\hline Bacteria identified- Wound culture & $6462-6$ & $459784(4.3)$ \\
\hline Antibiotic- Agar diffusion & $45187-2$ & $328113(3.0)$ \\
\hline Fungus Identified- Culture & $580-1$ & $291370(2.7)$ \\
\hline Mycobacterium sp identified & $543-9$ & $284631(2.6)$ \\
\hline Bacteria identified (Anaerobic+Aerobic)- Culture & $21020-3$ & $283577(2.6)$ \\
\hline Staphylococcus aureus methicillin resistant isolate- Culture & $13317-3$ & $207718(1.9)$ \\
\hline Bacteria identified- Body fluid culture & $611-4$ & $186589(1.7)$ \\
\hline Microscopic observation- Wet preparation & $680-9$ & $174113(1.6)$ \\
\hline Bacteria identified- Stool culture & $625-4$ & $159657(1.5)$ \\
\hline Chlamydia trachomatis + Neisseriagonorrhoeae- Probe & $36902-5$ & $155606(1.4)$ \\
\hline Bacteria identified- Aerobic culture (Wound) & $632-0$ & $145213(1.3)$ \\
\hline Bacteria identified- CSF culture & $606-4$ & $139418(1.3)$ \\
\hline Bacteria identified (Anaerobic)- Culture & $635-3$ & $112496(1.0)$ \\
\hline Bacteria identified (Aerobic)- Culture & $634-6$ & $92773(0.9)$ \\
\hline Streptococcus pyogenes Ag - EIA & $6558-1$ & $89173(0.8)$ \\
\hline Microscopic observation (Gram Stain) & $664-3$ & $81129(0.8)$ \\
\hline Bacteria identified- Culture (system- xxx) & $6463-4$ & $67118(0.6)$ \\
\hline Antibiotic- Minimum inhibitory concentration & $21070-8$ & $58891(0.5)$ \\
\hline Bacteria identified- Culture & $43408-4$ & $57084(0.5)$ \\
\hline Bacteria identified (Sputum)- Respiratory culture & $624-7$ & $56906(0.5)$ \\
\hline
\end{tabular}




\begin{tabular}{|l|l|l|}
\hline Clostridium difficile toxin A+B (Stool) & $34713-8$ & 55567 (0.5) \\
\hline Bacteria identified- Throat culture & $626-2$ & $50977(0.5)$ \\
\hline
\end{tabular}

For test orders, 805 unique LOINC codes were used. After excluding “OTH” and missing, values, LOINC codes to identify bacteria in blood (35.0\%), urine (15.2\%), respiratory specimens $(4.8 \%)$, or wounds (4.2\%), and to test antibiotic susceptibilities by agar diffusion (3.0\%), were the five most frequent laboratory orders reported (Table 2). Fourteen hundred twenty eight unique LOINC and 608 unique SNOMED CT codes were used to describe laboratory test results. For results with LOINC codes, the five most commonly reported tests were: bacteria identified by blood culture (12.2\%), microscopic observation of unspecified specimen by Gram stain (8.9\%), appearance of unspecified specimen (7.6\%), microorganism identified in unspecified specimen by culture $(7.5 \%)$, and bacteria identified by urine culture (6.6\%)(Table 3a). For results with SNOMED CT codes, a qualifier for antimicrobial susceptibility (10.4\%), Escherichia coli (10.4\%), a qualifier for bacterial sensitivity (9.9\%), Staphylococcus aureus (9.7\%), and a qualifier for non-reactive status (6.5\%) were the five most frequent codes (Table 3b).

Table 3a. Twenty five most common hospital-based laboratory result types reported to BioSense, 2011, with Logical Observation Identifiers Names and Codes (LOINC) codes

\begin{tabular}{|l|l|l|}
\hline LOINC Long Name & $\begin{array}{l}\text { LOINC } \\
\text { Code }\end{array}$ & $\begin{array}{l}\text { Test Results } \\
\text { N (\%) }\end{array}$ \\
\hline Bacteria identified in blood by culture & $600-7$ & $1128381(12.2)$ \\
\hline Microscopic observation in unspecified specimen by gram stain & $664-3$ & $823440(8.9)$ \\
\hline Appearance of unspecified specimen & $33511-7$ & $707650(7.6)$ \\
\hline Microorganism Identified in unspecified specimen by culture & $11475-1$ & $696652(7.5)$ \\
\hline Bacteria identified in urine by culture & $630-4$ & $611553(6.6)$ \\
\hline Specimen Source of unspecified specimen & $31208-2$ & $532232(5.7)$ \\
\hline Bacteria identified in unspecified specimen & $41741-$ & $271916(2.9)$ \\
& $0 *$ & \\
\hline Service comment & $8251-1$ & $176943(1.9)$ \\
\hline Bacteria identified in unspecified specimen by respiratory culture & $32355-0$ & $148069(1.6)$ \\
\hline Fungus identified in unspecified specimen by culture & $580-1$ & $140800(1.5)$ \\
\hline Bacteria identified in blood by aerobe culture & $17928-3$ & $127807(1.4)$ \\
\hline Microscopic observation in unspecified specimen by other stain & $11546-9$ & $115486(1.2)$ \\
\hline Bacteria identified in wound by aerobe culture & $632-0$ & $110500(1.2)$ \\
\hline Microorganism or agent Identified in unspecified specimen & $41852-5$ & $107766(1.2)$ \\
\hline Mycobacterium SP Identified in unspecified specimen by culture & $543-9$ & $106956(1.2)$ \\
\hline Bacteria identified in wound by culture & $6462-6$ & $103717(1.1)$ \\
\hline Bacteria identified in blood by anaerobe culture & $17934-1$ & $98539(1.1)$ \\
\hline Microscopic observation in wound by Gram stain & $10357-2$ & $98288(1.1)$ \\
\hline Microscopic observation in unspecified specimen by wet preparation & $680-9$ & $93901(1.0)$ \\
\hline Gentamycin susceptibility & $18928-2$ & $75789(0.8)$ \\
\hline Microscopic observation in unspecified specimen by acid fast stain & $11545-1$ & $70546(0.8)$ \\
\hline Trimethoprim + Sulfamethoxazole susceptibility & $18998-5$ & $68972(0.7)$ \\
\hline Microorganism identified in stool by culture & $625-4$ & $64295(0.7)$ \\
\hline
\end{tabular}


\begin{tabular}{|l}
\hline Levofloxacin susceptibility \\
\hline Ciprofloxacin susceptibility:
\end{tabular}

\begin{tabular}{|l|l|}
$20629-2$ & $60212(0.7)$ \\
$18906-8$ & $51448(0.6)$
\end{tabular}

*- LOINC code 41741-0 has been deprecated and superseded by LOINC code 23667-9.

\#- LOINC code for Service Comment terms indicates user-defined text.

Among the 608 unique SNOMED CT codes used to report laboratory test results, 498 (81.9\%) had corresponding LOINC codes reported. For the 108 SNOMED codes that did not have corresponding LOINC codes in the reported laboratory results, we found that 49 (45.4\%) of the SNOMED CT concepts could be associated with at least one LOINC code. The majority of SNOMED CT concepts matched to LOINC (47 of 49) were microorganism related, while one was an anatomical structure (urethra) and one was a system concept (blood). Among 49 SNOMED CT concepts that matched to LOINC codes, 27 concepts matched to more than one LOINC code, depending on other LOINC components including property, timing, system, scale, and method. Among 59 SNOMED CT concepts not identified in mapping to LOINC codes, 47 were for microorganisms, seven were for qualifiers, three were for anatomical structures, and two were for systems.

Table 3b. Twenty five most common hospital-based laboratory results types reported to BioSense, 2011, with Systemized Nomenclature of Medicine Clinical Terms (SNOMED CT) codes

\begin{tabular}{|l|l|l|}
\hline Observation Identifier (OBS) Text & $\begin{array}{l}\text { SNOMEDCT } \\
\text { Code }\end{array}$ & $\begin{array}{l}\text { Test Results N } \\
\text { (\%) }\end{array}$ \\
\hline Susceptible & 131196009 & $79465(10.4)$ \\
\hline Escherichia coli & 112283007 & $79255(10.4)$ \\
\hline Sensitive & 83185005 & $75142(9.9)$ \\
\hline Staphylococcus aureus & 3092008 & $74216(9.7)$ \\
\hline Non-Reactive & 131194007 & $49852(6.5)$ \\
\hline Resistant & 30714006 & $44504(5.8)$ \\
\hline None* & 260413007 & $39924(5.2)$ \\
\hline Pseudomonas aeruginosa & 52499004 & $30555(4.0)$ \\
\hline Gram-negative bacillus & 87172008 & $18016(2.4)$ \\
\hline Leukocyte & 52501007 & $17678(2.3)$ \\
\hline Staphylococcus, coagulase negative & 116197008 & $16094(2.1)$ \\
\hline Proteus mirabilis & 73457008 & $14522(1.9)$ \\
\hline Reactive & 11214006 & $13693(1.8)$ \\
\hline Klebsiella pneumoniae & 56415008 & $13446(1.8)$ \\
\hline Staphylococcus epidermidis & 60875001 & $13182(1.7)$ \\
\hline Enterococcus fecalis & 78065002 & $12610(1.7)$ \\
\hline Klebsiella pneumonia ss. pneumoniae & 18400002 & $9681(1.3)$ \\
\hline Yeast & 62093005 & $8457(1.1)$ \\
\hline Enterococcus species & 131297007 & $7733(1.0)$ \\
\hline Enterobacter cloacae & 14385002 & $6794(0.9)$ \\
\hline Enterococcus & 2785000 & $5720(0.8)$ \\
\hline Candida albicans & 53326005 & $5446(0.7)$ \\
\hline No organism seen & 27863008 & $5139(0.7)$ \\
\hline & &
\end{tabular}




\begin{tabular}{|l|l|l|}
\hline Staphylococcus species & 116499001 & $4362(0.6)$ \\
\hline Stenotrophomonas maltophilia & 113697002 & $4342(0.6)$ \\
\hline Streptococcus agalactiae & 43492007 & $4113(0.5)$ \\
\hline
\end{tabular}

*_ "None" is a SNOMED CT qualifier value for "absence findings"

\section{Discussion}

The hospitals included in our study used LOINC as the coding system to record laboratory test orders. Laboratory test results were coded using both LOINC and SNOMED CT coding systems, though, the use of LOINC was much more common. Our findings indicate that participating hospital laboratories undertook reporting of laboratory test orders and results as recommended in ELR HL7 messaging guidance [32]. However, missing or non-informative codes comprised almost a quarter of laboratory test orders and a third of test results from hospitals reporting to BioSense in 2011.

By design, the primary objectives of BioSense program were to monitor clinical syndromes related to infectious diseases for early outbreak detection and ongoing situational awareness [27-29]. Therefore, Biosense recommended that participating hospitals preferentially report laboratory information related to infectious diseases. As a result, the majority of the laboratory reports in this analysis were microbiology related. We examined both test orders and preliminary and final test results. Monitoring laboratory test orders could provide early warning signals of suspicion of infectious disease, while monitoring results could contribute to biosurveillance by providing increased diagnostic specificity to automated syndromic case definition algorithms based on chief complaint text fields [26,33-35]. Laboratory data could also be used to evaluate interventions, and to monitor disease trends and progression, which might result in timely more effective outbreak response and management $[1,13]$.

In ELR reporting, the debate over the definition of "questions" (orders) and "answers" (results) is far from over. It has been suggested that LOINC is used for coding laboratory "questions" while SNOMED CT is used for coding the "answers". [22] Laboratory reports from hospitals in our analysis indicate that LOINC codes are used to code laboratory orders as well as results, illustrating LOINC's potential to provide codes for "questions" as well as "answers". On the other hand, there may be unique situations where a SNOMED CT code is required in combination with a LOINC code to fully represent a lab test result. For example, the SNOMED CT system allows coding for certain clinical structures (cervix, urethra), and for certain conditions (e.g., hyperbilirubinemia), that are currently not accounted for in the LOINC system.

Several earlier reports have suggested different mapping strategies to associate laboratory codes to diseases or health conditions [20,36-41]. Our findings suggest that the BioSense surveillance program might be able to focus on a small subset of LOINC and SNOMED CT codes related to diseases or syndromes of interest, as evidenced by the use of a relatively small number of unique LOINC $(n=1,428)$ and SNOMED CT $(n=608)$ codes in the data we analyzed compared to the number of codes available. To determine if laboratory data improves syndromic surveillance performance for enhanced outbreak detection and improved situational awareness, an evaluation of syndromic case definitions that incorporate laboratory test order or result information is required.

Some limitations in interpreting our findings should be noted. Non-federal hospitals who participated in BioSense are not a representative sample of all the U.S. hospitals. Use of the convenience sample of facilities that are able and willing to share electronic laboratory data with CDC may limit the generalizability of our findings. Furthermore, laboratory data in this analysis were primarily collected 
for infectious disease syndromic surveillance purposes. Therefore our findings may not be representative of the use of LOINC and SNOMED CT codes for ELR for all hospital laboratory testing. Completeness of ELR data in BioSense has not been validated prior to this study, which shows that one-third of electronic laboratory records are missing standardized codes or are coded as HL7 null "OTH" for result information that would be necessary for interpretation for surveillance. We are unable to report specific accuracy metrics such as specificity or sensitivity related to LOINC and SNOMED CT due to lack of a reference "gold standard".

The current analysis has several important strengths, beginning with computability of the coded laboratory test order and result information in the HL7 messaging format that supports automated categorization for surveillance. Consequently, we were able to merge data files from different hospitals to create one large laboratory data repository and apply standard criteria that could potentially augment information from other surveillance data sources. Laboratory reports in this analysis derived from local hospitals illustrate the potential value of standardizing laboratory data to provide timely information to hospital based infection preventionists and local health departments for situation awareness and early response, as well as contributing to national biosurveillance. Most importantly this study adds value to existing scientific knowledge by describing the use of LOINC and SNOMED CT codes in ELR contributed by select U.S. hospitals as healthcare organizations move towards implementing Meaningful Use and health information exchange [42,43].

\section{Conclusion}

We analyzed more than 14 million laboratory reports from non-federal hospital to assess the use of two structured coding systems, SNOMED CT and LOINC. The LOINC system was used more commonly than SNOMED system in the hospitals studied. Missing data and differences in representing laboratory test orders and results may inhibit effective analysis of electronic laboratory data. Increased completeness of coded lab data, data management tools that translate locally coded laboratory test information into LOINC or SNOMED CT codes, and increased participation of hospitals laboratories are needed to fully realize the value of laboratory data in public health practice and syndromic surveillance.

\section{Acknowledgments}

We are thankful to all non-federal hospitals participating in the BioSense Program that contributed laboratory data used in this manuscript.

Contributorship Statement:

SD, SB, CW and SG contributed equally in conceptualizing, analyzing, and interpreting data. SD drafted the manuscript. All authors including AD, UA contributed in revising it critically for important intellectual content of the paper. All authors have approved the final version of the paper submitted for publication.

\section{Competing interest:}

There are no competing interests

\section{Funding:}

No external funding was received for this project. 
There are no additional published or unpublished data available from the study.

The findings and conclusions in this article ate those of the authors and do not represent the official position of the Centers for Disease Control and Prevention or the Department of Veterans Affairs.

\section{References:}

1. Kite-Powell AH. 2008. J J; Hopkins, R S. Potential effects of electronic laboratory reporting on improving timeliness of infectious disease notification--Florida, 2002-2006. MMWR Morb Mortal Wkly Rep. 57, 1325-28. PubMed

2. Moore KM, Reddy V, Kapell D, Balter S. 2008. Impact of electronic laboratory reporting on hepatitis A surveillance in New York City. J Public Health Manag Pract. 14, 437-41. PubMed http://dx.doi.org/10.1097/01.PHH.0000333877.78443.f0

3. Overhage JM, Grannis S, McDonald CJ. 2008. A comparison of the completeness and timeliness of automated electronic laboratory reporting and spontaneous reporting of notifiable conditions. Am J Public Health. 98, 344-50. PubMed http://dx.doi.org/10.2105/AJPH.2006.092700

4. Effler P, Ching-Lee M, Bogard A, Ieong MC, Nekomoto T, et al. 1999. Statewide system of electronic notifiable disease reporting from clinical laboratories: comparing automated reporting with conventional methods. JAMA. 282, 1845-50. PubMed http://dx.doi.org/10.1001/jama.282.19.1845

5. Sahm DF, Critchley IA, Kelly LJ, et al. 2001. Evaluation of current activities of fluoroquinolones against gram-negative bacilli using centralized in vitro testing and electronic surveillance. Antimicrob Agents Chemother. 45, 267-74. PubMed http://dx.doi.org/10.1128/AAC.45.1.267$\underline{274.2001}$

6. Stern L, Lightfoot D. 1999. Automated outbreak detection: a quantitative retrospective analysis. Epidemiol Infect. 122, 103-10. PubMed http://dx.doi.org/10.1017/S0950268898001939

7. Panackal AA, M'Ikanatha NM, Tsui FC, et al. 2002. Automatic electronic laboratory-based reporting of notifiable infectious diseases at a large health system. Emerg Infect Dis. 8, 685-91. PubMed http://dx.doi.org/10.3201/eid0807.010493

8. Overhage JM, Suico J, McDonald CJ. 2001. Electronic laboratory reporting: barriers, solutions and findings. J Public Health Manag Pract. 7, 60-66. PubMed http://dx.doi.org/10.1097/00124784$\underline{200107060-00007}$

9. Lazo R, Li W, Meigs M, et al. 2006. The APHL/CDC Public Health Laboratory Interoperability Project Portal: a web-based collaborative tool to establish a national harmonized vocabulary for public health data exchange. AMIA Annu Symp Proc. 2006, 999. PubMed

10. Miller RH, Sim I. 2004. Physicians' use of electronic medical records: barriers and solutions. Health Aff (Millwood). 23, 116-26. PubMed http://dx.doi.org/10.1377/hlthaff.23.2.116

11. McDonald CJ. 1997. The barriers to electronic medical record systems and how to overcome them. J Am Med Inform Assoc. 4, 213-21. PubMed http://dx.doi.org/10.1136/jamia.1997.0040213

12. McGinn CA, Gagnon MP, Shaw N, et al. 2012. Users' perspectives of key factors to implementing electronic health records in Canada: a Delphi study. BMC Med Inform Decis Mak. 12, 105. PubMed http://dx.doi.org/10.1186/1472-6947-12-105 
13. CDC. Electronic reporting of laboratory data for public health: meeting report and recommendations. Atlanta, GA: Centers for Disease Control and Prevention; 1997.

14. Jernigan DB. 2001. Electronic laboratory-based reporting: opportunities and challenges for surveillance. Emerg Infect Dis. 7, 538. PubMed http://dx.doi.org/10.3201/eid0707.017717

15. Boonstra A, Broekhuis M. 2010. Barriers to the acceptance of electronic medical records by physicians from systematic review to taxonomy and interventions. BMC Health Serv Res. 10, 231. PubMed http://dx.doi.org/10.1186/1472-6963-10-231

16. Dixon BE, McGowan JJ, Grannis SJ. Electronic laboratory data quality and the value of a health information exchange to support public health reporting processes. AMIA Annu Symp Proc 2011;2011:322-30.

17. Forrey AW, McDonald CJ, DeMoor G, et al. 1996. Logical observation identifier names and codes (LOINC) database: a public use set of codes and names for electronic reporting of clinical laboratory test results. Clin Chem. 42, 81-90. PubMed

18. Huff SM, Rocha RA, McDonald CJ, et al. 1998. Development of the Logical Observation Identifier Names and Codes (LOINC) vocabulary. J Am Med Inform Assoc. 5, 276-92. PubMed http://dx.doi.org/10.1136/jamia.1998.0050276

19. Khan AN, Griffith SP, Moore C, Russell D, Rosario AC, Jr, et al. 2006. Standardizing laboratory data by mapping to LOINC. $J$ Am Med Inform Assoc. 13, 353-55. PubMed http://dx.doi.org/10.1197/jamia.M1935

20. Kim H, El-Kareh R, Goel A, Vineet FNU, Chapman WW. 2012. An approach to improve LOINC mapping through augmentation of local test names. J Biomed Inform. 45, 651-57. PubMed http://dx.doi.org/10.1016/j.jbi.2011.12.004

21. Lin MC, Vreeman DJ, McDonald CJ, Huff SM. 2011. A characterization of local LOINC mapping for laboratory tests in three large institutions. Methods Inf Med. 50, 105-14. PubMed http://dx.doi.org/10.3414/ME09-01-0072

22. McDonald CJ, Huff SM, Suico JG, et al. 2003. LOINC, a universal standard for identifying laboratory observations: a 5-year update. Clin Chem. 49, 624-33. PubMed http://dx.doi.org/10.1373/49.4.624

23. Cornet R, de Keizer N. 2008. Forty years of SNOMED: a literature review. BMC Med Inform Decis Mak. 8(Suppl 1), S2. PubMed http://dx.doi.org/10.1186/1472-6947-8-S1-S2

24. Rodrigues JM, Kumar A, Bousquet C, Trombert B. 2009. Using the CEN/ISO standard for categorial structure to harmonise the development of WHO international terminologies. Stud Health Technol Inform. 150, 255-59. PubMed

25. Massey KA, Ansermino JM, von Dadelszen P, Morris TJ, Liston RM, et al. 2009. What is SNOMED CT ${ }^{\circledR}$ and Why Should the ISSHP Care? Hypertens Pregnancy. 28, 119-21. PubMed http://dx.doi.org/10.1080/10641950802601294

26. Asatryan A, Benoit S, Ma H, English R, Elkin P, et al. 2011. Detection of pneumonia using freetext radiology reports in the BioSense system. Int $J$ Med Inform. 80, 67-73. PubMed http://dx.doi.org/10.1016/j.ijmedinf.2010.10.013 
27. Benoit SR, Burkom H, McIntyre AF, et al. 2012. Pneumonia in US hospitalized patients with influenza-like illness: BioSense, 2007-2010. Epidemiol Infect. 141(4), 1-11. PubMed

28. Bradley CA, Rolka H, Walker D, Loonsk J. 2005. BioSense: implementation of a national early event detection and situational awareness system. MMWR Morb Mortal Wkly Rep. 54(Suppl), 1119. PubMed

29. Loonsk JW. 2004. BioSense--a national initiative for early detection and quantification of public health emergencies. MMWR Morb Mortal Wkly Rep. 53(Suppl), 53-55. PubMed

30. Tokars JI, English R, McMurray P, Rhodes B. 2010. Summary of data reported to CDC's national automated biosurveillance system, 2008. BMC Med Inform Decis Mak. 10, 30. PubMed http://dx.doi.org/10.1186/1472-6947-10-30

31. CDC. PHIN Messaging Guide for Syndromic Surveillance. CDC; 2011.

32. HL7 Version 2.5.1 Implementation guide: orders and observations; interoperable laboratory result reporting to EHR (US Realm), Release 1. Office of National Coordinator (ONC); 2007.

33. Public Health Systems and Emerging Infections: Assessing the Capabilities of the Public and Private Sectors: Workshop Summary. In: Davis JR, Lederberg J, eds. Public Health Systems and Emerging Infections: Assessing the Capabilities of the Public and Private Sectors: Workshop Summary. Washington (DC)2000.

34. Benoit SR, McDonald LC, English R, Tokars JI. 2011. Automated surveillance of Clostridium difficile infections using BioSense. Infect Control Hosp Epidemiol. 32, 26-33. PubMed http://dx.doi.org/10.1086/657633

35. Bravata DM, McDonald KM, Smith WM, et al. 2004. Systematic review: surveillance systems for early detection of bioterrorism-related diseases. Ann Intern Med. 140, 910-22. PubMed http://dx.doi.org/10.7326/0003-4819-140-11-200406010-00013

36. Abhyankar S, Demner-Fushman D, McDonald CJ. 2012. Standardizing clinical laboratory data for secondary use. J Biomed Inform. 45, 642-50. PubMed http://dx.doi.org/10.1016/j.jbi.2012.04.012

37. Fidahussein M, Friedlin J, Grannis S. Practical challenges in the secondary use of real-world data: the notifiable condition detector. AMIA Annu Symp Proc 2011;2011:402-8.

38. Gamache RE, Dixon BE, Grannis S, Vreeman DJ. Impact of selective mapping strategies on automated laboratory result notification to public health authorities. AMIA Annu Symp Proc 2012;2012:228-36.

39. Simpson CR, Anandan C, Fischbacher C, Lefevre K, Sheikh A. 2007. Will Systematized Nomenclature of Medicine-Clinical Terms improve our understanding of the disease burden posed by allergic disorders? Clin Exp Allergy. 37, 1586-93. PubMed http://dx.doi.org/10.1111/j.13652222.2007.02830.x

40. Vreeman DJ, McDonald CJ. 2006. A comparison of intelligent mapper and document similarity scores for mapping local radiology terms to LOINC. AMIA Annu Symp Proc. 2006, 809-13. PubMed

41. Zunner C, Burkle T, Prokosch HU, Ganslandt T. 2012. Mapping local laboratory interface terms to LOINC at a German university hospital using RELMA V.5: a semi-automated approach. J Am Med Inform Assoc. PubMed 
42. CMS. Medicare and Medicaid programs; electronic health record incentive program--stage 2. Final rule. Fed Regist 2012;77:53967-4162. PubMed

43. 2012. Office of the National Coordinator for Health Information T. Health information technology: revisions to the 2014 edition electronic health record certification criteria; and Medicare and Medicaid programs; revisions to the Electronic Health Record Incentive Program. Interim final rule with comment period. Fed Regist. 77, 72985-91. PubMed 